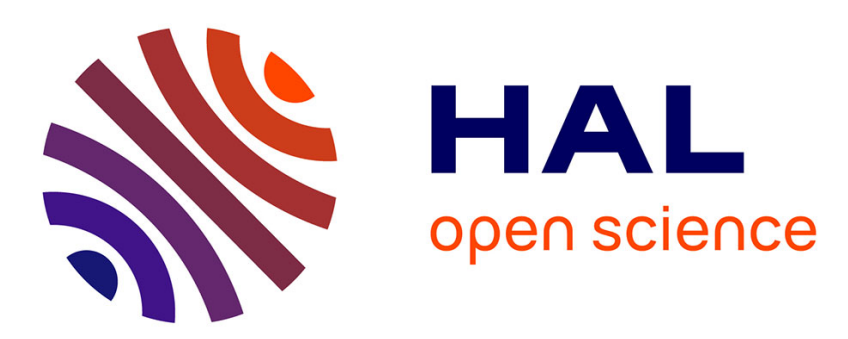

\title{
Influence de l'état mécanique d'un alliage réfractaire sur sa réactivité à haute température
}

\author{
B. Dionnet, F. Clemendot, E. Nardou
}

\section{To cite this version:}

B. Dionnet, F. Clemendot, E. Nardou. Influence de l'état mécanique d'un alliage réfractaire sur sa réactivité à haute température. Journal de Physique IV Proceedings, 1993, 03 (C9), pp.C9-963-C9970. 10.1051/jp4:1993999 . jpa-00252442

\section{HAL Id: jpa-00252442 https://hal.science/jpa-00252442}

Submitted on 1 Jan 1993

HAL is a multi-disciplinary open access archive for the deposit and dissemination of scientific research documents, whether they are published or not. The documents may come from teaching and research institutions in France or abroad, or from public or private research centers.
L'archive ouverte pluridisciplinaire HAL, est destinée au dépôt et à la diffusion de documents scientifiques de niveau recherche, publiés ou non, émanant des établissements d'enseignement et de recherche français ou étrangers, des laboratoires publics ou privés. 


\title{
Influence de l'état mécanique d'un alliage réfractaire sur sa réactivité à haute température
}

\author{
B. Dionnet $\left({ }^{1}\right)$, F. Clemendot $\left({ }^{2}\right)$ et F. Nardou $\left({ }^{1}\right)$
}

(1) Laboratoire de Céramiques Nouvelles, URA CNRS 320, 123, Avenue Albert Thomas, 87060 Limoges Cedex France

$\left({ }^{2}\right)$ EDF, Direction des Etudes et Recherches, Les Renardières, Route de Sens, Ecuelles, $\mathbf{7 7 2 5 0}$ Moret sur Loing France

\begin{abstract}
The aim of this work is the reactivity of a ferritic alloy studied considering both chemical and mechanical evolutions versus time between 800 and $1300{ }^{\circ} \mathrm{C}$ in air. A singular strength behaviour is showed at $900^{\circ} \mathrm{C}$ in isothermal conditions whereas an accelerated oxidation is visible at the same temperature. The evolution of the Young's modulus and thermal expansion studies explained these anomalies with carbides precipitation.
\end{abstract}

\section{Introduction.}

Les alliages ferritiques de type FeCrAl présentent de nombreuses applications industrielles, en particulier, la fabrication d'éléments chauffants. Néanmoins, ces aciers sont sensibles à l'oxydation à haute température et leur tenue à l'oxydation peut être modifiée en fonction de leur état métallurgique et mécanique.

L'étude présentée a pour objectif de corréler le comportement à l'oxydation d'un alliage industriel avec l'évolution des propriétés mécaniques à haute température.

\section{Conditions expérimentales.}

2.1 MATÉRIAU. - L'alliage étudié est le kanthal A1, diamètre 3,25 mm. La composition de cet acier ferritique est donnée tableau $\mathrm{I}$.

Tableau I. - Analyse chimique du kanthal A1 (\% poids).

\begin{tabular}{|c|c|c|c|c|c|c|c|c|c|}
\hline Fe & Cr & Al & Si & Ni & Mn & Zr & W & Cu & Co \\
\hline Bal. & 21,1 & 5,66 & $\mathbf{0 , 2 7}$ & 0,25 & 0,14 & 0,11 & 0,1 & 0,05 & $\mathbf{0 , 0 4}$ \\
\hline
\end{tabular}

\begin{tabular}{|c|c|c|c|c|c|c|c|c|}
\hline $\mathbf{C}$ & $\mathbf{V}$ & $\mathbf{M o}$ & $\mathbf{Y}$ & $\mathbf{N}$ & $\mathbf{P}$ & $\mathbf{M g}$ & $\mathbf{S}$ & $\mathbf{N b}$ \\
\hline 0,026 & 0,02 & $\leq 0,01$ & $<0,01$ & 0,009 & 0,008 & 0,006 & $<0,001$ & 0,0003 \\
\hline
\end{tabular}


Les échantillons prélevés directement sur la bobine de fil possèdent un rayon de courbure voisin de $32 \mathrm{~cm}$. Avant les essais, ils ne subissent aucune déformation mécanique ni traitement thermique supplémentaires. Ils sont simplement dégraissés.

2.2 DisPOSITIFS EXPÉRIMENTAUX. - L'oxydation isotherme des échantillons (longueur = $15 \mathrm{~mm}$ ) est suivie entre 800 et $1300^{\circ} \mathrm{C}$ sous débit d'air à la pression atmosphérique avec une microbalance Setaram. Des travaux antérieurs [1] décrivent le protocole suivi pour enregistrer le gain de masse en fonction du temps pour chaque température. La morphologie de la couche d'oxyde est étudiée au microscope électronique à balayage. La nature du produit de la réaction est déterminée par microanalyse $\mathrm{X}$ en dispersion d'énergie et par diffraction des rayons $\mathrm{X}$ avec une anticathode de chrome.

Les éprouvettes de traction, de longueur initiale entre repères $\left(L_{\mathrm{o}}\right)$ égale à $19,5 \mathrm{~mm}$, présentent à leurs extrémités des calottes sphériques élaborées par fusion à l'arc électrique sous argon pour la fixation aux tiges assurant la transmission de la force. Le dispositif expérimental est constitué d'une machine d'essais mécaniques de type Wolpert TZZ régulée en force [2]. Lors de la montée en température $\left(10^{\circ} \mathrm{C} / \mathrm{mn}\right)$ l'échantillon est maintenu sous argon très pur et n'est soumis à aucune force extérieure. Il est nécessaire de compenser les dilatations thermiques du système en régulant à force nulle. L'atmosphère d'essai (débit d'argon ou air) est choisie lorsque la température de palier est atteinte. Après un maintien de 0 à 24 heures à force nulle, l'éprouvette subit un essai de traction avec une vitesse de sollicitation égale à $5 \mathrm{~mm} / \mathrm{mn}$. La force et l'allongement sont alors mesurés.

L'étude dilatométrique du kanthal $\mathrm{Al}$ a été réalisée sur un dilatomètre différentiel Netzsch.

La détermination du module d'Young unidirectionnel a été réalisée à l'ENSCI de Limoges par méthode échographique ultrasonore à haute température. La mesure du temps de propagation $(t)$ de l'onde dans l'échantillon permet de calculer la vitesse de l'onde longitudinale $\left(V_{\mathrm{L}}\right)$ puis d'en déduire la valeur du module d'Young $(E)$ connaissant la masse volumique du matériau $(\rho)[3]$. Il est nécessaire de tenir compte de l'allongement de l'échantillon en fonction de la température.

$$
\begin{gathered}
V_{\mathrm{L}}=2 \cdot L / t \\
E=\rho \cdot V_{\mathrm{L}}^{2}
\end{gathered}
$$

\section{Résultats.}

3.1 CARACTÉRISATION MÉCANIQUe. - La résistance à la rupture conventionnelle $R_{\mathrm{m}}$ et l'allongement à rupture $A \%$ obtenus lors des essais de traction entre 900 à $1200^{\circ} \mathrm{C}$ sous air et sous argon sont regroupés figures 1 et 2 . Chacune de ces valeurs correspond à la moyenne d'au moins 3 essais réalisés dans des conditions identiques.

A $900{ }^{\circ} \mathrm{C}$, la résistance à la rupture passe par un minima après une heure de maintien en température, puis voit sa valeur croître. Cette augmentation du $R_{\mathrm{m}}$ est attribuable à l'augmentation de la taille des grains qui ont de plus en plus de mal à s'orienter dans le sens de la sollicitation. Ce phénomène semble plus marqué sous argon. Inversement l'allongement à la rupture diminue.

A haute température ce phénomène de grossissement de grain n'influe plus sur les propriétés mécaniques car les grains s'accommodent plastiquement lors de la traction. 


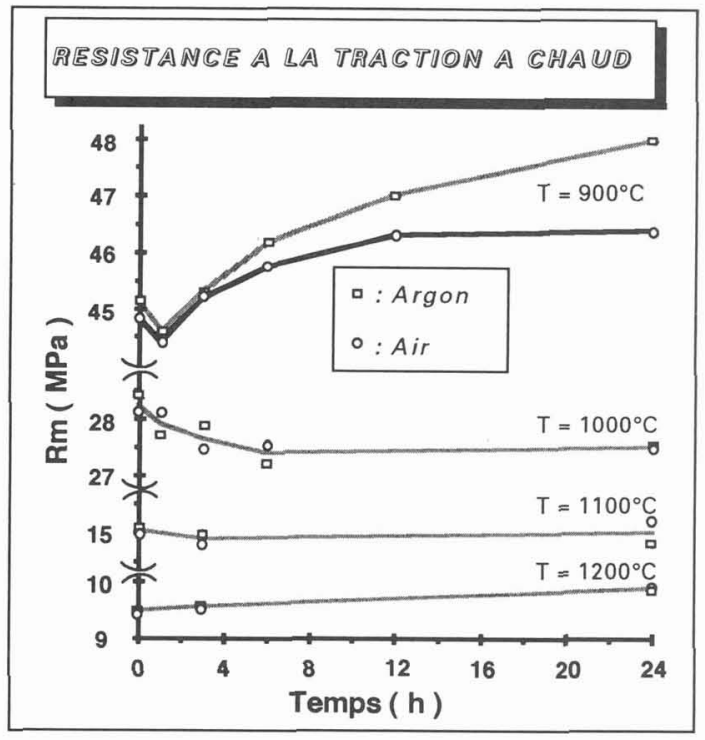

Fig. 1

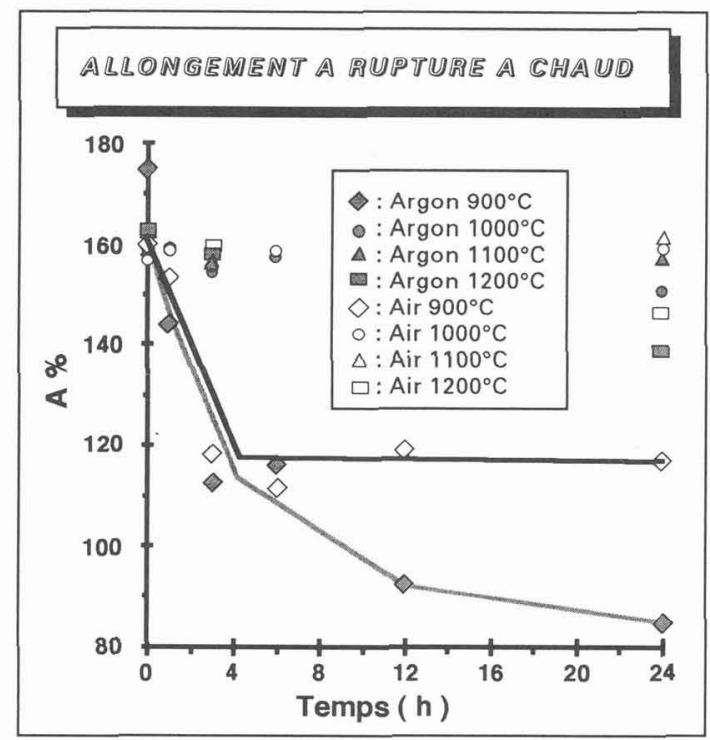

Fig. 2

Fig. 1. - Résistance à la rupture du kanthal Al en fonction du temps de maintien en température. Fig. 2. - Allongement à la rupture du kanthal $\mathrm{Al}$ en fonction du temps de maintien en température.

Un comportement particulier a également été observé lors de la mesure du module d'Young à $900^{\circ} \mathrm{C}$ après une montée en température identique à l'essai de traction (Fig. 3). Le module d'élasticité présente à cette température un maximum qui semble coïncider avec le minimum de la résistance à la rupture. Cependant, l'évolution observée par cette méthode échographique ultrasonore n'est pas attribuable à la croissance des grains.

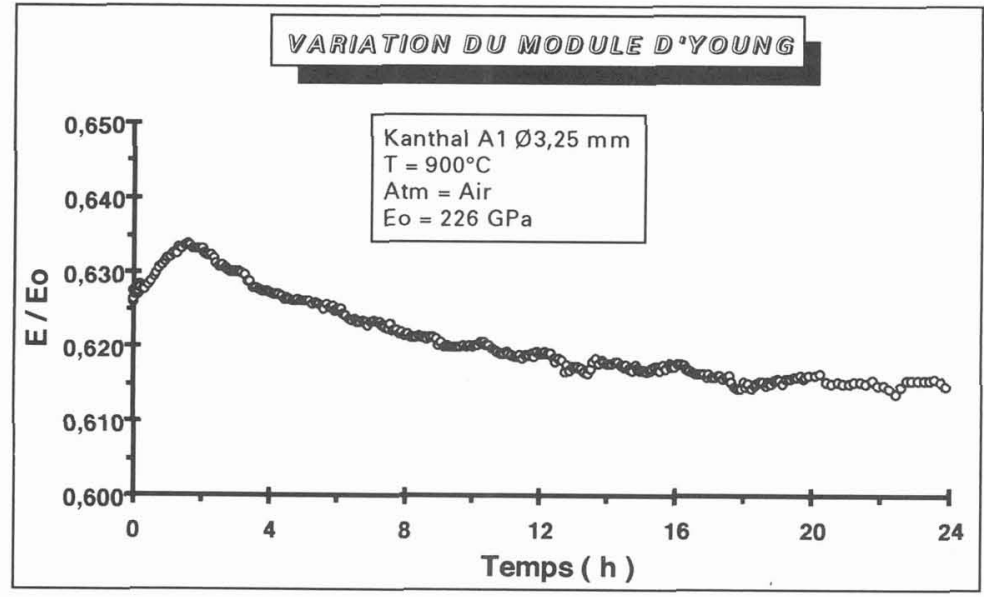

Fig. 3. - Variation du module d'Young à $900^{\circ} \mathrm{C}$ en fonction du temps. 
3.2 CARACTÉRISATION STRUCTURALE. - L'analyse radiocristallographique de la surface des échantillons met en évidence la présence majoritaire d'alumine alpha (Fiche 10-0173 du fichier ASTM) pour les températures supérieures et égales à $900^{\circ} \mathrm{C}$. A $800^{\circ} \mathrm{C}$ l'intensité des pics de diffraction est trop faible pour identifier avec certitude les phases en présence.

L'étude de la surface après 24 heures d'oxydation isotherme montre une évolution de la morphologie de la couche formée (Tableau II). Les produits de réaction épousent, et même amplifient, les défauts observés sur le matériau initial qui est très tourmenté et fortement écroui. Aux basses températures $\left(800\right.$ et $\left.900{ }^{\circ} \mathrm{C}\right)$, il y a croissance d'oxyde lamellaire, probablement du fait de l'accommodation des contraintes de croissance (Fig. 4a). A haute température, l'oxyde est majoritairement granulaire (Fig. 4b) sauf au voisinage de zones fortement contraintes (écaillage, fissures, faible rayon de courbure) où apparaissent des lamelles d'oxyde. Pour les températures intermédiaires $\left(0,5\right.$ à $0,6 T_{\mathrm{f}}$; avec $T_{\mathrm{f}}=2050{ }^{\circ} \mathrm{C}$ pour $\alpha \mathrm{Al}_{2} \mathrm{O}_{3}$ ) l'oxyde n'a pas un comportement purement élastique ou plastique, ce qui se traduit en terme morphologique par la présence d'oxyde d'aspects variables, lamellaire jusqu'à prendre la forme de "rose des sables" (Fig. 4c) ou granulaire jusqu'à l'aspect en forme de "nids d'abeilles" (Fig. 4d).

Tableau II. - Morphologie des couches après 24 heures sous air.

\begin{tabular}{|c|c|c|c|c|c|c|}
\hline Morphologie $/ T\left({ }^{\circ} \mathrm{C}\right)$ & 800 & 900 & 1000 & 1100 & 1200 & 1300 \\
\hline lamellaire & +++ & +++ & ++ & ++ & + & + \\
\hline roses des sables & & & ++ & & & \\
\hline nids d'abeilles & & & & ++ & & \\
\hline granulaire & & & ++ & ++ & +++ & +++ \\
\hline
\end{tabular}

L'observation au microscope optique des sections polies transversales après oxydation isotherme de $\mathbf{2 4}$ heures montre une épaisseur d'oxyde qui n'est pas parfaitement régulière sur toute la périphérie du métal. Globalement l'épaisseur de la couche d'oxyde augmente avec la température, passant de 1 à $8 \mu \mathrm{m}$ entre 800 et $1300^{\circ} \mathrm{C}$. A $900^{\circ} \mathrm{C}$ l'épaisseur de la couche est supérieure à celle obtenue à 1000 et $1100^{\circ} \mathrm{C}$ conformément au gain de masse enregistré (Chap. 3.3).

Les microanalyses $\mathrm{X}$ montrent l'existence d'inclusions de types oxydes, carbures ou mixtes dans la matrice métallique avant et après oxydation. Les carbures mis en évidence sont constitués principalement de zirconium et parfois de titane. Les oxydes sont de nature complexe avec l'yttrium comme élément majeur. Le calcium, l'arsenic et bien d'autres impuretés peuvent également être présentes. Quelle que soit la température de traitement la couche d'oxyde est constituée principalement d'aluminium et d'oxygène.

3.3 GinétiQue D'OXYDATION. - En première approximation il est possible, du fait de la faible épaisseur d'oxyde par rapport au métal, de représenter le gain de masse par unité de surface en fonction du temps d'oxydation (Fig. 5). Le gain de masse enregistré à $900^{\circ} \mathrm{C}$ est supérieur à ceux mesurés à 1000 et $1100^{\circ} \mathrm{C}$. Cette anomalie est similaire à celle rencontrée dans d'autres travaux $[4,5]$. Lors des premières heures d'oxydation les courbes ne sont pas homothétiques. Globalement la couche formée présente un caractère protecteur. 


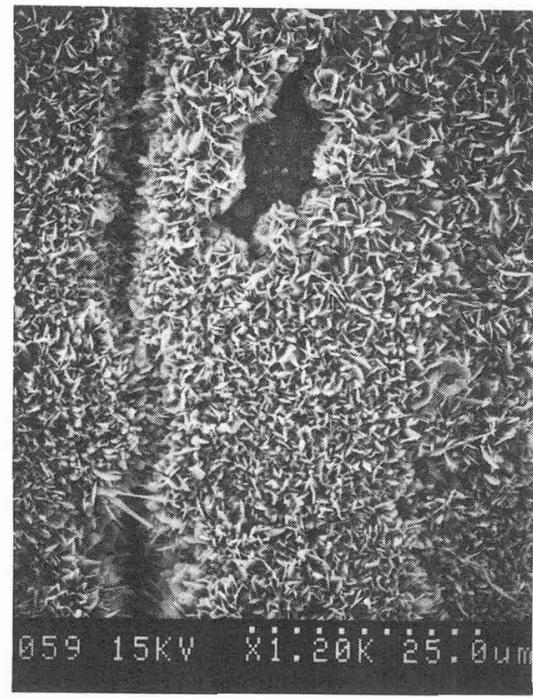

a)

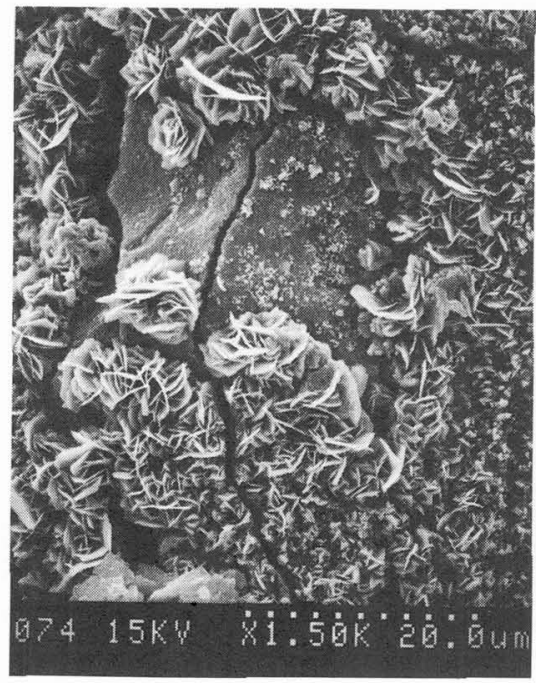

c)

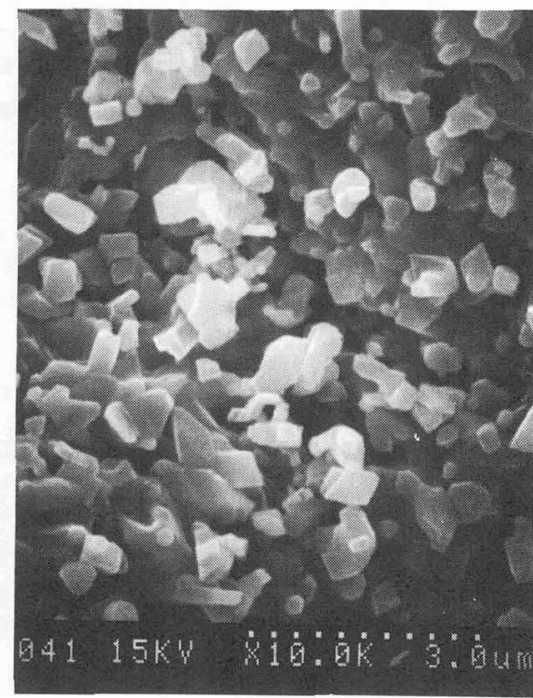

b)

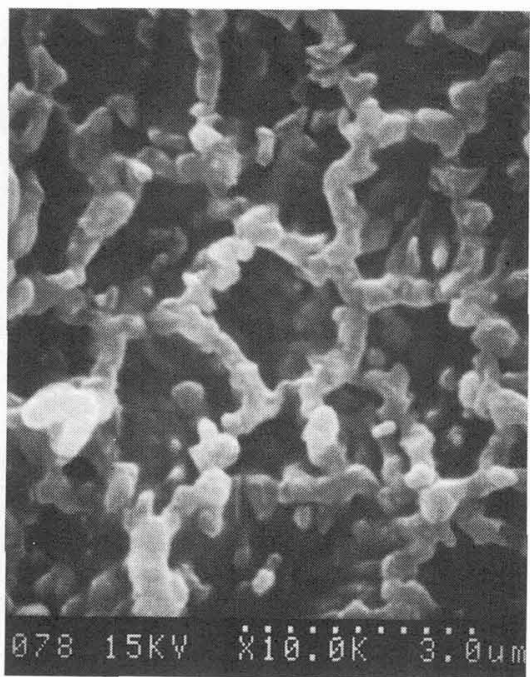

d)

Fig. 4. - Morphologie de la surface oxydée du kanthal Al après 24 heures de traitement isotherme: a) $T=900^{\circ} \mathrm{C}$; b) $T=1300^{\circ} \mathrm{C}$; c) $T=1000^{\circ} \mathrm{C}$; d) $T=1100^{\circ} \mathrm{C}$.

En accord avec les observations effectuées sur la couche d'oxyde, nous pouvons admettre que celle-ci est constituée majoritairement d'alumine alpha et relier l'avancement de la réaction aux constantes de vitesse relatives aux processus d'interface externe, d'interface interne et de diffusion selon la relation de Billy et Valensi en symétrie cylindrique [6]. Les transformées relatives au processus diffusionnel permettent d'accéder aux constantes de vitesse du tableau III.

L'évolution de ces constantes selon la loi d'Arrhenius pour les températures supérieures 


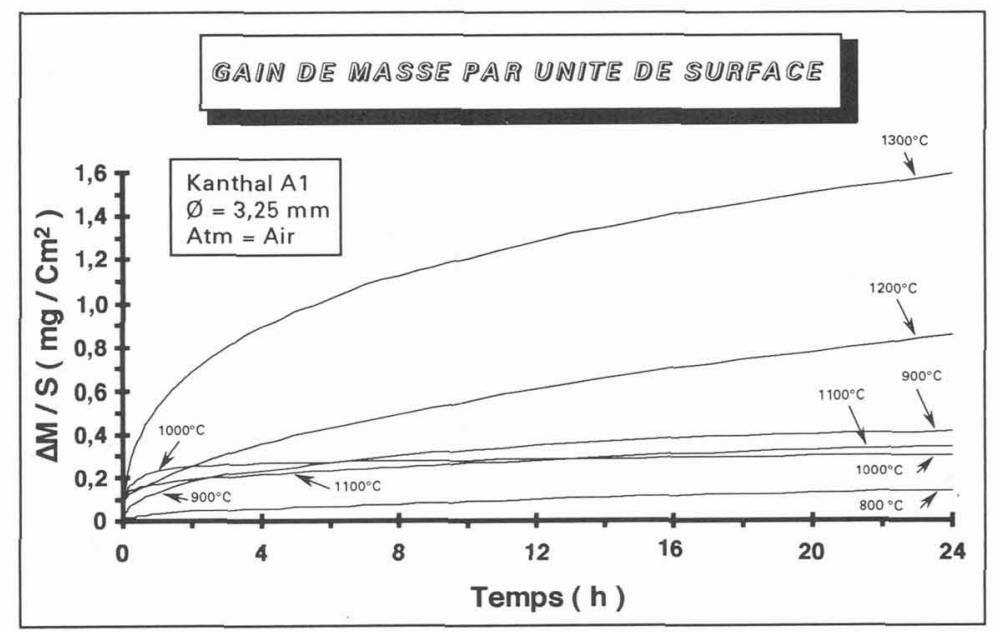

Fig. 5. - Gain de masse par unité de surface du kanthal Al en fonction du temps d'oxydation isotherme sous air.

Tableau III. - Valeurs des constantes de diffusion.

\begin{tabular}{|c|c|c|c|c|}
\hline $\begin{array}{c}\text { Temp. } \\
\left({ }^{\circ} \mathrm{C}\right)\end{array}$ & $\begin{array}{c}\text { Temps } \\
{\left[t_{\min }-t_{\max }\right]} \\
(\text { heures })\end{array}$ & $\begin{array}{c}K_{\mathbf{D}} \\
{[1 \mathrm{E}-13]} \\
(\mathrm{eq} /(\mathrm{cm} . \mathrm{s}))\left({ }^{*}\right)\end{array}$ & $\begin{array}{c}\text { Erreur type sur } K_{\mathbf{D}} \\
{[1 \mathrm{E}-13]} \\
(\mathrm{eq} /(\mathrm{cm} . \mathrm{s}))\left({ }^{*}\right)\end{array}$ & $\begin{array}{c}\text { Coeff. } \\
\text { de } \\
\text { corrélation }\end{array}$ \\
\hline 800 & 0 à 24 & 6,38265 & 0,06950 & 0,9974 \\
\hline 900 & 3 à 15 & 66,7435 & 1,19735 & 0,9971 \\
\hline 1000 & 5 à 24 & 8,16941 & 0,07621 & 0,9990 \\
\hline 1100 & 2 à 22 & 32,0959 & 0,35308 & 0,9983 \\
\hline 1200 & 0 à 24 & 242,882 & 0,36143 & 0,9999 \\
\hline 1300 & 6 à 22 & 687,716 & 11,4394 & 0,9973 \\
\hline
\end{tabular}

$\left({ }^{*}\right)$ eq $=$ equivalent $[6]$.

ou égales à $1000^{\circ} \mathrm{C}$ correspond à une énergie d'activation de $255 \pm 20 \mathrm{~kJ} \cdot \mathrm{mol}^{-1}$ (Fig. 6). En revanche, on note l'existence de deux points singuliers $\left(800^{\circ} \mathrm{C}\right.$ et $\left.900^{\circ} \mathrm{C}\right)$ bien que ceux-ci évoluent avec la même énergie d'activation $\left(245 \mathrm{~kJ} \cdot \mathrm{mol}^{-1}\right)$.

La valeur de l'énergie d'activation à haute température est proche de celle trouvée par Oishi et Kingery [7] relative à l'énergie $(241 \mathrm{~kJ} / \mathrm{mol})$ d'activation de diffusion de l'oxygène dans l'alumine monocristalline $\left(T<1500^{\circ} \mathrm{C}\right)$. Cette valeur a été interprétée par les auteurs comme étant caractéristique d'une diffusion extrinsèque due soit aux impuretés, soit aux phénomènes de court circuits. 


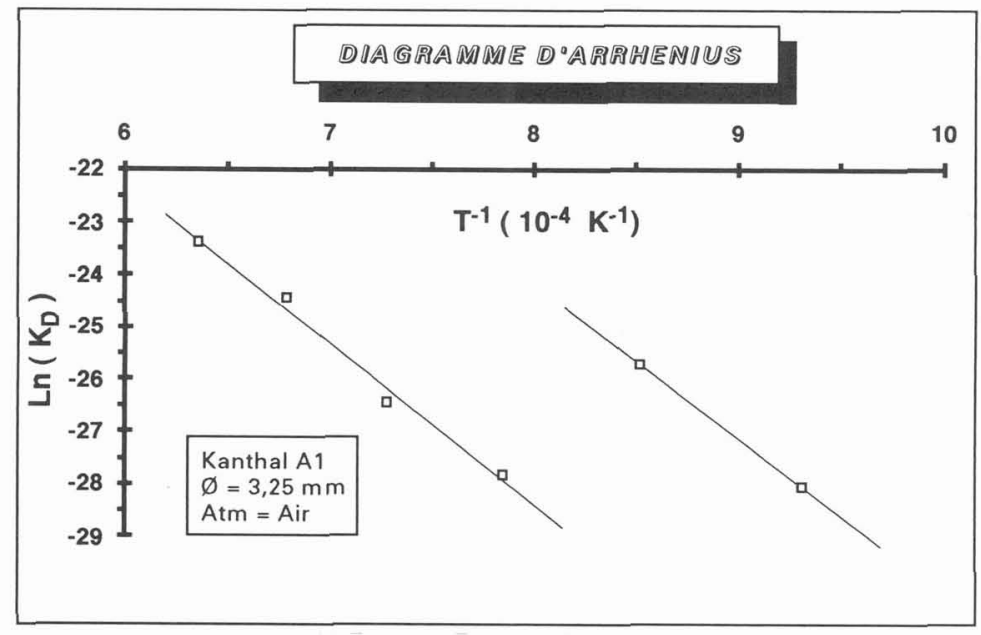

Fig. 6. - Variation logarithmique, en fonction de l'inverse de la température des constantes de diffusion.

\section{Discussion.}

L'étude de l'oxydation isotherme du kanthal $\mathrm{Al}$, diamètre $3,25 \mathrm{~mm}$, sous air pendant 24 heures confirme la présence d'un régime de diffusion activé thermiquement à haute température, avec formation d'une couche majoritaire d'alumine $\alpha$. La morphologie de la couche d'oxyde est alors granulaire. A plus basse température $\left(800\right.$ et $\left.900^{\circ} \mathrm{C}\right)$ la croissance de l'oxyde se fait sous forme de lamelles. L'accommodation des contraintes de croissance peut s'effectuer de plusieurs manières en fonction de la température de traitement, soit par écaillage et fissuration, soit en générant un oxyde lamellaire, soit encore plastiquement lorsque la température est suffisante. Néanmoins, à $900^{\circ} \mathrm{C}$ il apparaît après 2 heures une augmentation de la vitesse d'oxydation. Ce comportement anormal peut trouver une explication dans l'évolution mécanique du matériau à la même température. Par ailleurs des essais dilatométriques (Fig. 7) réalisés en condition non isotherme puis isotherme sous air à $1350^{\circ} \mathrm{C}$ ont permis de mettre en évidence la précipitation de carbures à $900^{\circ} \mathrm{C}(\mathrm{Zr}, \mathrm{Cr}, \mathrm{Ti}, \ldots)$ en accord avec d'autres auteurs [5].

En corrélant les résultats dilatométriques, mécaniques et cinétiques, il apparait possible d'attribuer l'augmentation du module d'Young à la précipitation de phases ayant des modules d'élasticité supérieurs à celui de la matrice initiale. Le module mesuré donnant une valeur globale sur l'ensemble de l'échantillon on doit admettre que l'évolution inverse notée après environ 2 heures (instant où précisément on observe l'accélération cinétique de l'oxydation) correspond à une évolution de ces précipités.

La précipitation de phase(s) secondaire(s) qui crée des défauts au sein de la matrice métallique pourrait expliquer la légère diminution de la résistance à la traction à $900{ }^{\circ} \mathrm{C}$, au tout début du traitement thermique pendant lequel est susceptible d'intervenir un phénomène de recristallisation de l'alliage.

Ces interprétations sont en accord avec les observations faites sur un alliage industriel recuit à $1200^{\circ} \mathrm{C}$ (mise en solution solide du carbone) puis oxydé à $900^{\circ} \mathrm{C}[5,8]$. L'accélération de l'oxydation notée à cette température avait été interprétée par les auteurs en terme de 


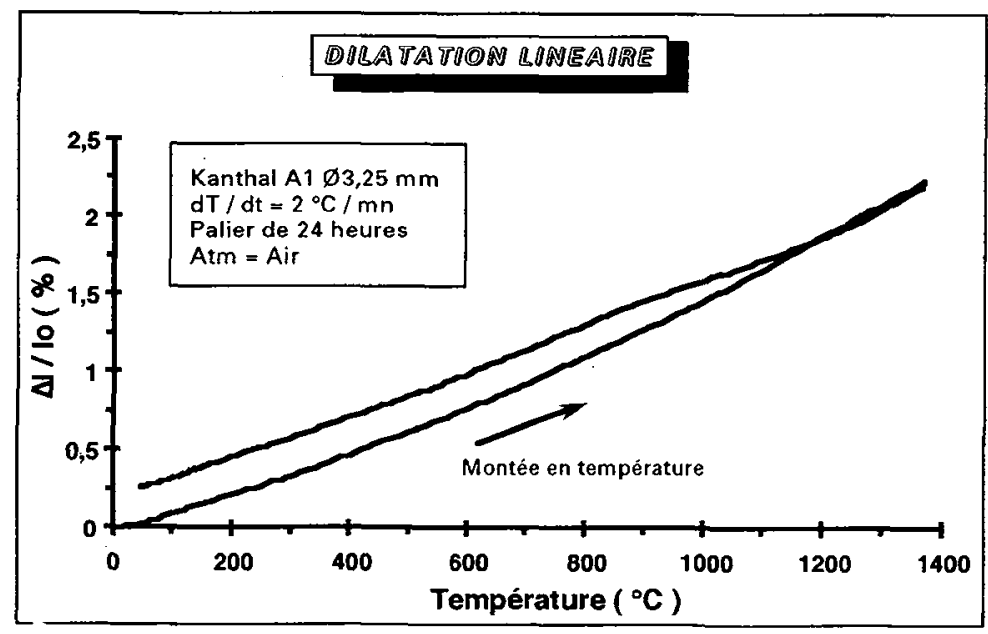

Fig. 7. - Dilatation linéaire du kanthal A1.

compétition entre l'oxydation et la précipitation des carbures. Ces derniers se formeraient aléatoirement dans la matrice et dans la zone interne de la couche d'oxyde en cours de croissance. Ces précipités seraient des chemins préférentiels de diffusion, à l'origine de l'accélération de l'oxydation à $900^{\circ} \mathrm{C}$.

D'une manière générale la nature et l'état chimique initial des impuretés de constitution comme le carbone mais aussi, $\mathrm{Zr}, \mathrm{Mn}, \mathrm{Y}$, et bien d'autres jouent un rôle important sur le comportement à l'oxydation des alliages. Nous avons montré par ce travail que le comportement à l'oxydation des alliages ne pouvait être dissocié du comportement mécanique du matériau et que les deux évolutions physico-chimique et mécanique devaient être considérées simultanément au cours d'une étude d'oxydation.

\section{Bibliographie}

[1] RanaIVONIARIVO L., Thèse (Limoges, 1985).

[2] GNASSE DAGo L., Thèse (Limoges, 1988).

[3] Huger M., Thèse (Limoges, 1992).

[4] Hearl A., Gulbransen F., Kenneth F., Andrew, J. Electrochem. Soc. 106 (1959) 294.

[5] BEN ABDERRAZIK G., Thèse (Paris Sud, 1986).

[6] Billy M., Valensi G., J. Chem. Phys. 53 (1956) 832.

[7] Oishi Y., Kingery W.D., J. Chem. Phys. 33 (2) (1960) 480.

[8] Ben AbDerrazik G., Moulin G., HunTz A.M., Berneron R., J. Mat. Sci. 19 (1984) 3173. 